\title{
Características epidemiológicas e espaço-temporal de casos novos de hanseníase em municípios do estado de Alagoas, Brasil
}

\author{
Epidemiological and spatio-temporal characteristics of new leprosy cases in municipalities in the \\ state of Alagoas, Brazil \\ Características epidemiológicas y espacio-temporales de nuevos casos de lepra en municipios del \\ estado de Alagoas, Brasil
}

\section{Resumo}

Introdução: As dificuldades de enfrentamento da hanseníase reafirmam a importância de estudos epidemiológicos em diferentes localidades para melhor avaliar a magnitude de sua endemicidade. Objetivo: analisar a distribuição dos casos novos e coeficientes de detecção da hanseníase no estado de Alagoas. Método: Trata-se de um estudo ecológico. Foram incluídos os casos de hanseníase notificados em 102 municípios do estado de Alagoas. O estudo foi baseado em registros obtidos no Sistema Nacional de Notificação, a partir da Secretaria Estadual de Saúde (2010-2019) e por dados demográficos do censo populacional (2010). Empregou-se análise de associação, espacial e de tendência temporal. Resultados: Foram notificados 4.343 casos de hanseníase nos municípios. A taxa média de detecção de casos novos para a população total foi estimada em 10,85/100.000 habitantes, variando de 12,57 (2010) a 11,63 (2019). A maior taxa foi estimada para o município de Santana do Ipanema (58,2/100.000 habitantes), considerado como hiperendêmico. Taxas com valores de muita alta endemia foram observadas em 13 municípios. Houve tendência de redução do número médio de casos em 2010-2014 (APC = -4,34, IC95\%: -10,5-2.3) e de aumento em 2015-2019 (APC $=3,04$, IC95\%: -15,1-25). As taxas brutas e bayesiana demonstraram muitos municípios com característica de alta endemia, nas regiões oeste, noroeste, nordeste e sul. Conclusão: Este estudo evidenciou clusters em áreas de maior vulnerabilidade socioeconômica. Recomenda-se o planejamento de ações preventivas, busca ativa de casos e avaliação de contatos nas áreas identificadas.

Palavras-chave: Hanseníase; Epidemiologia; Análise espaço-temporal.

\section{Abstract}

Introduction: The difficulties in coping with leprosy reaffirm the importance of epidemiological studies in different locations to better assess the magnitude of its endemicity. Objective: to analyze the distribution of new cases and leprosy detection coefficients in the state of Alagoas. Method: This is an ecological study. Leprosy cases reported in the municipalities of Alagoas were included. The study was based on records obtained in the National Notification 
System, from State Department of Health (2010-2019) and demographic data from the population census (2010). Association, spatial and trend temporal analysis were used. Results: 4,343 cases of leprosy were reported in the municipalities. The average rate of detection of new cases for the total population was estimated at 10.85 , ranging from 12.57 (2010) to 11.63 (2019). The highest rate was estimated for the municipality of Santana do Ipanema (58.2), considered to be hyper-endemic. Rates with very high endemic values were observed in 13 municipalities. There was a downward trend in the mean number of cases in 2010-2014 (APC $=-4.34, \mathrm{CI} 95 \%$ : -10.5-2.3) and an increase in 2015-2019 (APC $=3.04$, CI95\%: $-15.1-25)$. The gross and Bayesian rates showed many municipalities with a high endemic characteristic, in the west, northwest, northeast and south regions. Conclusion: This study showed clusters in areas of greater socioeconomic vulnerability. It is recommended to plan preventive actions, to actively search for cases and to evaluate contacts in the identified areas.

Keywords: Leprosy; Epidemiology; Spatio-temporal analysis.

\section{Resumen}

Introducción: Las dificultades para hacer frente a la lepra reafirman la importancia de los estudios epidemiológicos en diferentes localizaciones para evaluar mejor la magnitud de su endemicidad. Objetivo: analizar la distribución de nuevos casos y coeficientes de detección de lepra en el estado de Alagoas. Método: Este es un estudio ecológico. Se incluyeron los casos de lepra notificados en los municipios de Alagoas. El estudio se basó en registros obtenidos en el Sistema Nacional de Notificación, del Departamento de Salud del Estado (2010-2019) y datos demográficos del censo de población (2010). Se utilizaron análisis de asociación, espacial y de tendencias temporales. Resultados: Se notificaron 4.343 casos de lepra en los municipios. La tasa promedio de detección de nuevos casos promedio para la población total se estimó en 10.85, variando de 12.57 (2010) a 11.63 (2019). La tasa más alta se estimó para el municipio de Santana do Ipanema (58.2), considerado hiperendémico. 2Se observaron tasas con valores endémicos muy altos en 13 municipios. Hubo una tendencia a la baja en el número promedio de casos en 2010-2014 (APC = 4.34, IC95\%: -10,5-2.3) y un aumento en 2015-2019 (APC = 3.04, IC95\%: -15,1-25). Las tasas brutas y bayesianas mostraron muchos municipios con una alta característica endémica, en las regiones oeste, noroeste, noreste y sur. Conclusión: Este estudio mostró conglomerados en áreas de mayor vulnerabilidad socioeconómica. Se recomienda planificar acciones preventivas, buscar activamente casos y evaluar contactos en las áreas identificadas.

Palabras clave: Lepra; Epidemiología; Análisis espacio-temporal.

\section{Introdução}

A hanseníase é uma doença infectocontagiosa, causada por Mycobacterium leprae (M. leprae), que provoca grave comprometimento neurológico, quando não diagnosticada e tratada adequadamente (Wilder-Smith \& Brakel, 2008; Talhari, Talhari \& Penna, 2015). Os danos neurais causados pela doença podem levar à perda sensitiva, amiotrofia, feridas e deformidades na face, mãos e pés, que em alguns casos são irreversíveis (Wilder-Smith \& Brakel, 2008). Estas complicações podem ocorrer antes do diagnóstico, durante a terapia multidroga (MDT) ou após o término do tratamento (Lockwood \& Saunderson, 2012), e são acentuadas por fatores como o diagnóstico tardio, precárias condições socioeconômicas e estigma para com os doentes acometidos pela doença (Withington, et al., 2013; Leon, et al., 2016).

Em 2019, foram registrados 202.185 casos de hanseníase no mundo, dos quais cerca de 13,8\% (27.863) ocorreram no Brasil (WHO, 2020). A taxa de detecção de casos novos (TDCN) foi estimada em 11.2/100.000 habitantes para a população geral, o que caracteriza uma área de alta endemia (Brasil, 2021). Embora a detecção de casos de hanseníase entre os menores de 15 anos tenha apresentado uma tendência discreta de redução e/ou manutenção nos últimos anos (Schneider, et al., 2018), ainda foram notificados 1.868 casos novos (6,7\% dos casos totais) neste mesmo ano (Brasil, 2019). A taxa de detecção de casos de hanseníase na população infantil refere-se à infecção na comunidade e, portanto, demonstra a presença de transmissão ativa e de baixa efetividade dos programas de controle (Brasil, 2016).

A existência de aglomerados (clusters) de casos novos de hanseníase distribuídos heterogeneamente no Brasil evidencia a manutenção de elevada carga da doença em determinadas áreas (Freitas, Duarte \& Garcia, 2012). Os casos ocorrem com maior frequência nas regiões Norte, Centro-Oeste e Nordeste, que correspondem àquelas com os piores indicadores socioeconômicos do país (Penna, Oliveira \& Penna, 2009). O estado de Alagoas, localizado na região Nordeste do país, apresentou alta endemia de hanseníase (TDCN estimada em 6,95/100.000 habitantes, em 2019) e uma heterogeneidade na frequência dos casos em seus municípios, com importante morbidade pela doença em algumas microrregiões (Brasil, 2021). 
As dificuldades de enfrentamento e de eliminação reafirmam o caráter negligenciado da hanseníase e a configuram como um importante problema de saúde pública, uma vez que muitas características de seus determinantes ainda não são bem conhecidas (Visschedijk, et al., 2000). Uma melhor compreensão do perfil epidemiológico da hanseníase no estado de Alagoas ajudará a quantificar a carga da doença na população e a avaliar a magnitude de sua endemicidade. Nesse sentido, o objetivo deste trabalho foi analisar a distribuição dos casos novos e taxas de detecção da hanseníase no estado de Alagoas, no período de 2010 a 2019.

\section{Metodologia}

\section{Desenho de estudo e fontes dos dados}

Trata-se de um estudo ecológico, tendo como unidades de análise os 102 municípios do estado de Alagoas, região Nordeste do país, e seus respectivos casos notificados de hanseníase. Alagoas possui uma população estimada em 3.351.543 habitantes e é considerado um estado de grandes desigualdades socioeconômicas, apresentando o menor Índice de Desenvolvimento Humano (IDH) do país (0,631) e índice de Gini de 0,711 (IBGE, 2019).

O estudo foi baseado em: i) registros de hanseníase obtidos no Sistema Nacional de Notificação de Doenças do Ministério da Saúde (SINAN-hanseníase) (2010-2019) e ii) dados demográficos do censo populacional do Instituto Brasileiro de Geografia e Estatística (IBGE) (2010). Os dados do SINAN-hanseníase foram obtidos do banco de dados na Secretaria Estadual de Saúde do Estado de Alagoas (SESAU) e incluiu registros de todos os casos novos de hanseníase detectados entre $1^{\circ}$ de janeiro de 2010 e 31 de dezembro de 2019.

\section{Variáveis}

Foram consideradas variáveis demográficas e socioeconômicas, tais como sexo, raça/cor auto reportada, escolaridade e zona de residencial (rural, urbana e periurbana). As variáveis clínicas dos casos incluíram as formas clínicas (indeterminada, tuberculóide, borderline, virchowiana e não identificada), grau de deficiência física no momento do diagnóstico (grau 0, grau 1, grau 2 e não avaliado), modo de entrada (caso novo, transferência, recidiva e outros), modo de deteç̧ão (encaminhamento, demanda espontânea, exame de coletividade, exame de contato, e outros), baciloscopia (positiva, negativa e não realizada), e esquema terapêutico da poliquimioterapia (PQT) de acordo com a classificação operacional paucibacilar (PB) ou multibacilar (MB) (PQT/PB/6 doses, PQT/MB/12 doses, ou outros substitutivos).

\section{Análise dos dados}

As TDCN da hanseníase por 100.000 habitantes (casos/100.000 hab) foram estimadas para todos os municípios de 2010 a 2019. A suavização dessas taxas foi realizada para minimizar flutuações aleatórias, produzidas pelos pequenos números, mediante aplicação do método bayesiano empírico global (Bailey \& Gatrell, 1995). Para isso, foi obtida uma média ponderada entre o valor medido e a taxa média de vizinhança, com pesos inversamente proporcionais à população de cada área (Almeida, 2012). As taxas de deteç̧ão médias e as taxas suavizadas pela análise bayesiana foram apresentados em mapas temáticos, estratificados por quinquênios (2010-2014 e 2015-2019). A classificação dos municípios em relação à magnitude das TDCN foi feita de acordo com os Indicadores de Monitoramento do Progresso da Eliminação da Hanseníase enquanto problema de saúde pública, do Ministério da Saúde: TDCN >40,0/100.000 hab - hiperendemia; 20,00 a 39,99/100.000 hab muito alta endemia; 10,00 a 19,99/100.000 hab - alta endemia; 2,00 a 9,99/100.000 hab - média endemia; e <2,00/100.000 hab - baixa endemia (Brasil, 2016).

A normalidade e homocedasticidade das médias dos números de casos e médias das TDCN foram avaliadas pelos testes Lillierfors e Levene, respectivamente. Tendo em vista que não foram atendidos os pressupostos de normalidade e 
homocedasticidade, foi utilizado o teste não paramétrico de Kruskal Wallis. As comparações múltiplas foram feitas usando o teste de Dunn's. Já para as comparações das TDCN, após os testes de normalidade e homocedasticidade, foi utilizado o teste de Friedman. As análises foram realizadas para a população total.

As tendências no número de casos de hanseníase por ano foram estimadas usando modelos Joinpoint, a partir de um número mínimo de ponto que aumentam ou diminuem ao longo do período de tempo, observando-se as mudanças das tendências nos pontos de tempo. Foi calculada também a variação percentual anual (Annual Percentage Change - APC) e seu intervalo de confiança de 95\% (IC 95\%). A análise foi realizada utilizando o software Joinpoint Regression (Statistical Methodology and Applications Branch, Surveillance Research Program do National Cancer Institute, versão 4.7.0.0, National Cancer Institute, EUA).

Os mapas de análise espacial foram construídos a partir da base cartográfica do estado de Alagoas, disponível em fonte eletrônica do IBGE. Os dados georreferenciados foram analisados por meio do TerraView (Instituto Nacional de Pesquisas Espaciais (INPE), SP, BR 4.2.2) e Open Source Geospatial Foundation (QGIS) da OSGeo, CHI, USA, Versão 2.18.2). A existência de autocorrelação espacial para o subgrupo de indivíduos menores de 15 anos foi avaliada nos quinquênios 2010-2014 e 2015-2019 pelo cálculo do Índice Global de Moran, a partir de valores que variaram de -1 a 1 . Os índices positivos apontam para a existência de autocorrelação espacial entre municípios vizinhos; valores negativos indicam nenhuma semelhança entre eles; e índices próximos de zero significam nenhuma correlação espacial (Almeida, 2012; Anselin, 2005).

Uma análise descritiva, incluindo frequências absolutas e relativas foi realizada para investigar a distribuição das variáveis de características demográficas, socioeconômicas e clínicas, estratificados pela classificação operacional do caso. As diferenças de proporções entre as categorias de exposição foram analisadas utilizando o teste chi-quadrado de Pearson, considerando o nível de significância de 5\% e por meio do software Bioestat versão 0.5 .

\section{Aspectos éticos}

O estudo foi aprovado pelo Comitê de Ética em Pesquisa da Universidade de Ciências da Saúde do Estado de Alagoas parecer $n^{\circ}$ 3.228-221/CAAE 08754819.4.0000.5011.

\section{Resultados}

Entre os anos de 2010 e 2019 foram notificados 4.343 casos de hanseníase nos 102 municípios do estado de Alagoas. A TDCN média para a população total foi estimada em 10,85/100.000 habitantes, variando de 12,57/100,000 hab em 2010 a 11,63/100,000 hab em 2019. A maior TDCN média foi estimada para o município de Santana do Ipanema (58,2/100.000 hab), considerado como hiperendêmico. TDCN com valores de muita alta endemia foram observadas em 13 municípios, variando de 20,3/100.000 habitantes em Maragogi (localizado na microrregião do litoral norte alagoano e inserido na mesorregião do leste alagoano) até 35,2/100.000 habitantes em Barra de São Miguel (localizado na microrregião de Maceió e inserido na mesorregião do leste alagoano) (dados não mostrados). Foi observada tendência de redução do número médio de casos de hanseníase para o quinquênio 2010-2014, com APC estimada em -4,34 (IC95\%: -10,5-2.3). Já no quinquênio 2015-2019, o número de casos apresenta tendência de aumento e APC de 3,04 (IC95\%: -15,1-25) (Figura 1). 
Figura 1. Tendência temporal do número de casos de hanseníase nos 102 municípios de Alagoas para os quinquênios 20102016 e 2016-2019.

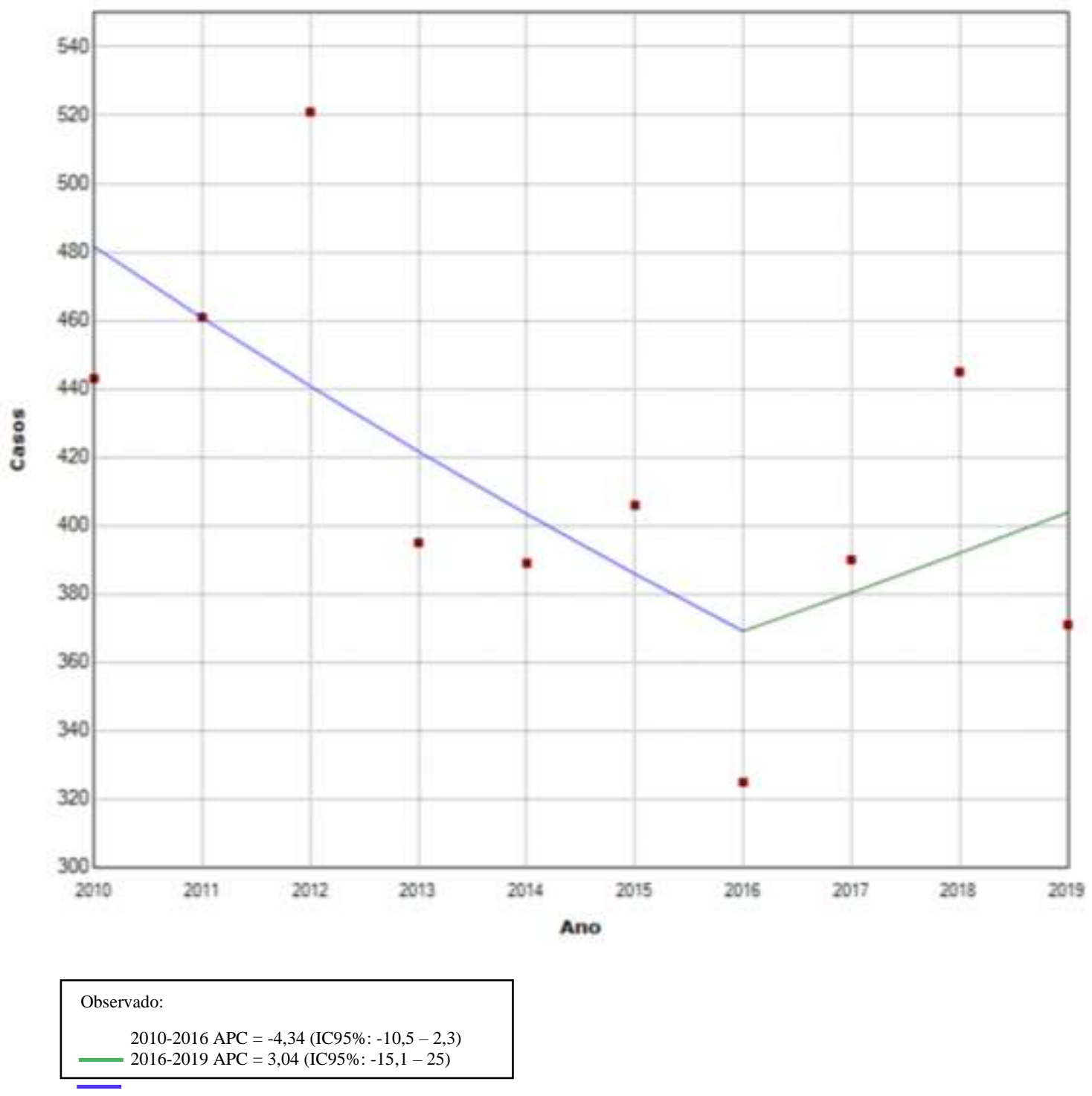

Fonte: Autores.

As taxas de deteç̧ão brutas foram espacialmente heterogêneas dentro do estado em todos os anos do período avaliado, com muito municípios com valores zero, sobretudo no ano de 2016 (Figura 2-1). As taxas suavizadas demonstram muitos municípios com alta e muito alta endemia entre os anos de 2010 e 2012 (Figura 2-2). Municípios com baixas taxas de detecção estavam próximos de outros com altas taxas de detecção. Foi observada concentração de municípios com taxas e superendemia entres as regiões oeste e noroeste do estado em todos os anos (Figura 2-2). Para o subgrupo de indivíduos com idade superior a 15 anos, foi observado padrão de distribuição espacial similar para os quinquênios 2010-2014 e 2015-2019, mas sem dependência espacial estatisticamente significante, com I =-0,033 ( $p=0,36)$ e $I=-0,026(p=0,39)$, respectivamente. 
Figura 2. Taxas de incidência brutas (1) e taxas bayesianas empíricas locais (2) de casos de hanseníase nos municípios de Alagoas para os quinquênios 2010-2014 e 2015-2019.

(1)

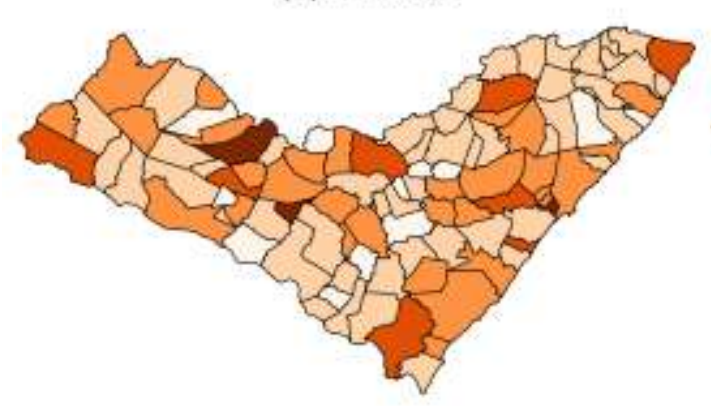

(2)

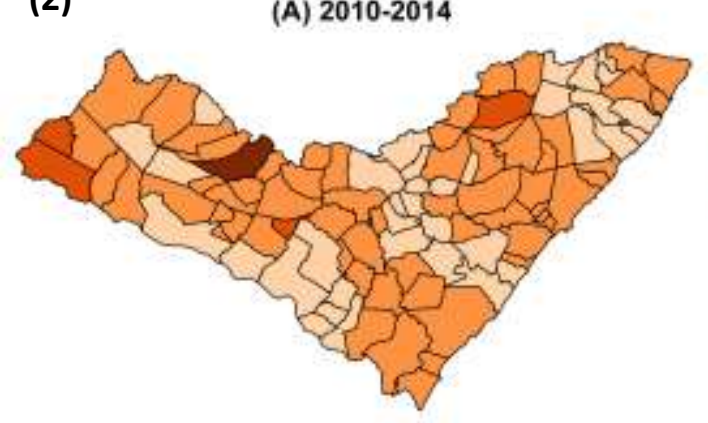

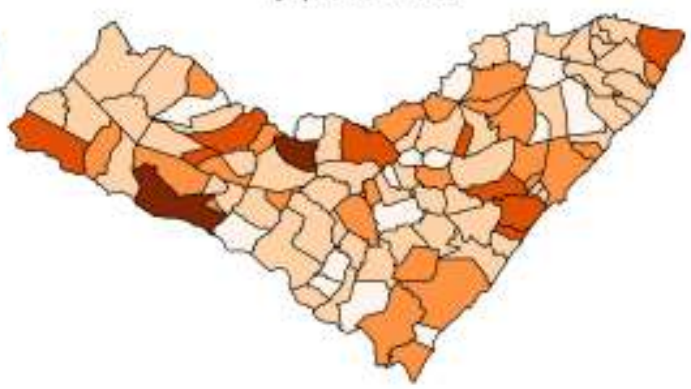

(B) 2015-2019

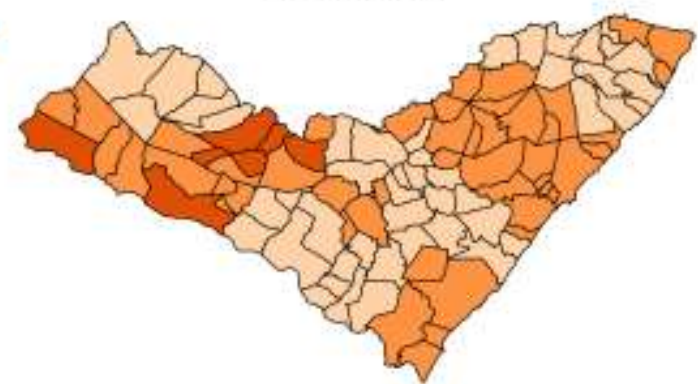

\section{Análise bayesiana}

Taxa local

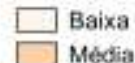

$\square$ Médii:

Alta

Muito alta

Hiperendèmica

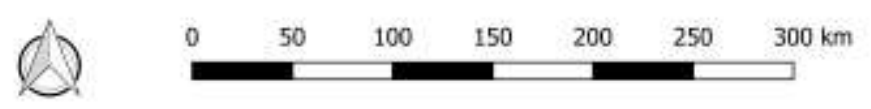

Fonte: Autores.

A maior parte dos casos era do sexo feminino (50,2\%), de raça/cor parda (67,5\%), com baixa escolaridade (primeiro grau incompleto 66,1\%) e residente em zonas urbanas (82,9\%). Os casos foram diagnosticados com forma clínica indeterminada (19,3\%), tuberculóide $(21,1 \%)$, dimorfa $(26,5 \%)$ e virchowiana $(16,2 \%)$, com grau $0(56,1 \%)$, grau $1(25,8 \%)$ e grau $2(8,3 \%)$ de incapacidade física. A maior parte dos casos foi notificada como casos novos (86,4\%), detectada a partir de encaminhamentos $(55,4 \%)$ e por demanda espontânea $(34,1 \%), 64,8 \%$ não realizaram baciloscopia e 57,1\% tiveram como esquema terapêutico a PQT/MB/12 doses (Tabela 1).

A hanseníase MB foi mais prevalente entre pessoas do sexo masculino (71,6\%), de raças/cores branca $(54,1 \%)$, preta $(62,9 \%)$, parda $(57,6 \%)$ e indígena $(64,0 \%)$, com primeiro grau incompleto $(59,0 \%)$ e completo $(55,3 \%)$, e entre todas as áreas de residência $(56,7 \%$ urbana, 58,5\% rural e 57,8\% periurbana), comparada à hanseníase PB. Os casos MB foram em sua maioria diagnosticados com formas clínicas dimorfa (98,5\%) e virchowiana (99,8\%), e com grau 1 (72,2\%) ou grau 2 (92,6\%) de incapacidade física. O modo detecção dos casos MB foi, na maioria das vezes, por encaminhamento (59,7\%) e por demanda espontânea (52,9\%), com esquema terapêutico de PQT/MB/12 doses $(99,4 \%)$ (Tabela 1). 
Tabela 1. Características sociodemográficas e clínicas dos casos de hanseníase diagnosticados em municípios de Alagoas, 2010-2019.

\begin{tabular}{|c|c|c|c|c|c|c|c|}
\hline \multirow{3}{*}{ Variáveis } & & & \multicolumn{4}{|c|}{ Classificação Operacional } & \multirow{3}{*}{$p$ valor $^{3}$} \\
\hline & \multicolumn{2}{|c|}{ Total } & \multicolumn{2}{|c|}{$\begin{array}{c}\text { PB } \\
(n=1.851)\end{array}$} & \multicolumn{2}{|c|}{$\begin{array}{c}\text { MB } \\
(\mathrm{n}=2.492)\end{array}$} & \\
\hline & $\mathbf{n}$ & $\%^{1}$ & $\mathbf{n}$ & $\%^{2}$ & $\mathbf{n}$ & $\%^{2}$ & \\
\hline Sexo & & & & & & & 0,001 \\
\hline Masculino & 2162 & 49,8 & 614 & 28,4 & 1548 & 71,6 & \\
\hline Feminino & 2181 & 50,2 & 1237 & 56,7 & 944 & 43,3 & \\
\hline Raça/Cor & & & & & & & 0,014 \\
\hline Branca & 732 & 17,3 & 336 & 45,9 & 396 & 54,1 & \\
\hline Preta & 572 & 13,5 & 212 & 37,1 & 360 & 62,9 & \\
\hline Amarela & 42 & 1,0 & 22 & 52,4 & 20 & 47,6 & \\
\hline Parda & 2852 & 67,5 & 1210 & 42,4 & 1642 & 57,6 & \\
\hline Indígena & 25 & 0,7 & 9 & 36,0 & 16 & 64,0 & \\
\hline Escolaridade & & & & & & & $<0,001$ \\
\hline Primeiro grau incompleto & 1826 & 66,1 & 748 & 41,0 & 1078 & 59,0 & \\
\hline Primeiro grau completo & 179 & 6,5 & 80 & 44,7 & 99 & 55,3 & \\
\hline Ensino médio incompleto & 228 & 8,3 & 126 & 55,3 & 102 & 44,7 & \\
\hline Ensino médio completo & 380 & 13,8 & 203 & 53,4 & 177 & 46,6 & \\
\hline Ensino superior incompleto & 64 & 2,3 & 43 & 67,2 & 21 & 32,6 & \\
\hline Ensino superior completo & 84 & 3,0 & 54 & 64,3 & 30 & 35,7 & \\
\hline Zona & & & & & & & 0,689 \\
\hline Urbana & 3394 & 82,9 & 1470 & 43,3 & 1924 & 56,7 & \\
\hline Rural & 653 & 16,0 & 271 & 41,5 & 382 & 58,5 & \\
\hline Periurbana & 45 & 1,1 & 19 & 42,2 & 26 & 57,8 & \\
\hline Forma clínica & & & & & & & $<0,001$ \\
\hline Indeterminada & 774 & 19,3 & 682 & 88,1 & 92 & 11,9 & \\
\hline Tuberculóide & 846 & 21,1 & 761 & 90,0 & 85 & 10,0 & \\
\hline Dimorfa & 1061 & 26,5 & 16 & 1,5 & 1045 & 98,5 & \\
\hline Virchowiana & 651 & 16,2 & 1 & 0,2 & 650 & 99,8 & \\
\hline Não identificado & 677 & 16,9 & 250 & 36,9 & 427 & 63,1 & \\
\hline Grau de incapacidade física no diagnóstico & & & & & & & $<0,001$ \\
\hline Grau 0 & 2282 & 56,1 & 1283 & 56,2 & 999 & 43,8 & \\
\hline Grau 1 & 1049 & 25,8 & 292 & 27,8 & 757 & 72,2 & \\
\hline Grau 2 & 338 & 8,3 & 25 & 7,4 & 313 & 92,6 & \\
\hline Não avaliado & 399 & 9,8 & 154 & 38,6 & 245 & 61,4 & \\
\hline Modo de Entrada & & & & & & & $<0,001$ \\
\hline Caso novo & 3749 & 86,4 & 1696 & 45,2 & 2053 & 54,8 & \\
\hline Transf. mesmo município & 78 & 1,8 & 32 & 41,0 & 46 & 59,0 & \\
\hline Transf. outro município & 96 & 2,2 & 25 & 26,0 & 71 & 74,0 & \\
\hline Transf. outro estado & 67 & 1,5 & 10 & 14,9 & 57 & 85,1 & \\
\hline Transf. outro país & 3 & 0,1 & 0 & 0,0 & 3 & 100,0 & \\
\hline Recidiva & 173 & 4,0 & 43 & 24,9 & 130 & 75,1 & \\
\hline Outros & 173 & 4,0 & 43 & 24,9 & 130 & 75,1 & \\
\hline Modo detecção do caso novo & & & & & & & $<0,001$ \\
\hline Encaminhamento & 2095 & 55,4 & 845 & 40,3 & 1250 & 59,7 & \\
\hline Demanda espontânea & 1291 & 34,1 & 608 & 47,1 & 683 & 52,9 & \\
\hline Exame de coletividade & 135 & 3,6 & 87 & 64,4 & 48 & 25,6 & \\
\hline Exame contato & 186 & 4,9 & 126 & 67,7 & 60 & 32,3 & \\
\hline Outros modos & 77 & 2,0 & 28 & 36,4 & 49 & 63,6 & \\
\hline Baciloscopia & & & & & & & $<0,001$ \\
\hline Positiva & 606 & 6,5 & 127 & 21,0 & 479 & 79,0 & \\
\hline Negativa & 684 & 18,7 & 305 & 44,6 & 379 & 55,4 & \\
\hline Não realizada & 2372 & 64,8 & 1150 & 48,5 & 1222 & 51,5 & \\
\hline Esquema terapêutico & & & & & & & $<0,001$ \\
\hline $\mathrm{PQT} / \mathrm{PB} / 6$ doses & 1839 & 42,5 & 1823 & 99,1 & 16 & 0,9 & \\
\hline $\mathrm{PQT} / \mathrm{MB} / 12$ doses & 2471 & 57,1 & 14 & 0,6 & 2457 & 99,4 & \\
\hline Outros esquemas substitutos & 21 & 0,5 & 6 & 28,6 & 15 & 71,4 & \\
\hline
\end{tabular}

${ }^{1}$ Percentual por coluna.

${ }^{2}$ Percentual por linha.

${ }^{3}$ Teste qui-quadrado de Pearson para verificar diferenças entre as categorias das variáveis de exposição.

Fonte: Autores. 


\section{Discussão}

A TDCN média do estado de Alagoas entre os anos de 2010 e 2019 foi semelhante às médias nacionais estimadas neste período (Brasil, 2021). Contudo, a distribuição espacial da hanseníase foi heterogênea, com clusters de altas TDCN nas regiões oeste e noroeste, que correspondem à mesorregião Sertão e Agreste alagoano. Muitos municípios apresentaram áreas de alta endemia e hiperendemia, como o município de Santana do Ipanema (localizado na microrregião de Santana do Ipanema e inserido na mesorregião do sertão alagoano) que apresentou uma TDCN cinco vezes maior que a média do estado. As razões para o agrupamento espacial das taxas de hanseníase podem estar na distribuição heterogênea de fatores como pobreza, desigualdade social, piores condições do domicílio e dificuldade ou falta de acesso aos serviços de saúde observadas no estado. Além disso, o perfil dos casos detectados no período demonstrou maiores proporções de hanseníase multibacilar, entre os quais foram também observados maior comprometimento físico (grau de incapacidade 1 ou 2) e maiores desvantagens socioeconômicas.

A hanseníase abrange um processo saúde-doença muito heterogêneo e, consequentemente, a análise da desigualdade e determinantes sociais envolvidos é também extremante complexa (WHO, 2010). A relação entre a hanseníase e as condições sociais e econômicas vem sendo tema importante de estudos nas últimas décadas e a literatura é bastante consistente sobre a determinação da pobreza na ocorrência e transmissão da hanseníase (Kerr-Pontes, et al., 2006; Monteiro, et al., 2017; Nery, et al., 2018; Pescarini, et al., 2019; Pescarini et al., 2020). A investigação recente de mais de 33 milhões de indivíduos no Brasil demonstrou que níveis mais baixos de renda familiar e condições desfavoráveis de vida familiar estavam associados a uma taxa geral de detecção de hanseníase até duas vezes maior (Nery, et al., 2019).

Essa relação entre altas taxas de detecção de hanseníase em áreas de baixo nível socioeconômico encontrada neste estudo é coerente com os resultados encontrados em alguns estudos realizados para outras áreas no Brasil. Um estudo realizado em Pernambuco (2005-2014) (Barbosa, et al., 2018), estado vizinho a Alagoas, estimou uma TDCN média de 21,88/100.000 hab em regiões do Agreste e Sertão pernambucanos, que variaram de 0,88/100.000 hab em Jurema a 85,0/100 000 hab em Trindade. Nestas áreas, o mapa Moran identificou 20 municípios com alta prioridade de atenção à hanseníase (Barbosa, et al., 2018). Já em São Paulo, entre os anos de 2009 e 2012, foi observado municípios com altas TDCN no mapa temático (8,65/100.000 hab e mais), localizados na região oeste do estado, na região metropolitana da capital e em alguns municípios do Vale do Paraíba (Ferreira \& Nascimento, 2019). As maiores TDCN identificadas em algumas regiões podem ser, em parte, explicadas pelo crescimento descontrolado da população associado aos movimentos populacionais (procedentes do interior do estado e/ou de outras regiões do país, principalmente Norte-Nordeste), sem melhora da estrutura urbana e condições de vida dessas populações (Murto, et al., 2013; Murto, et al., 2014). No Tocantins, a sobreposição geográfica de aglomerados de municípios de alto risco para a hanseníase refletiu a vulnerabilidade social das populações afetadas (Monteiro, et al., 2015). Os altos valores desses indicadores podem ser resultado da expansão geográfica e do processo de urbanização observados no estado, que podem facilitar a manutenção e disseminação da doença na região (Monteiro, et al., 2015).

Alguns estudos já demonstraram consistentes correlações entre urbanização e taxas mais altas de hanseníase (Pescarini, et al., 2018). O movimento migratório, comum em áreas urbanas, apresenta-se como um aspecto importante para o aumento da carga de hanseníase em centros urbanos. De modo geral, as condições de vida e moradia precárias, e a busca por postos de trabalho, caracterizam a migração como um processo de reprodução de pobreza e doenças (Murto, et al., 2013; Murto, et al., 2014). Este processo ocorre quando migrantes suscetíveis chegam em áreas de alta endemicidade e quando migrantes infectados passam para áreas não endêmicas, especialmente entre indivíduos provenientes de classes sociais desfavorecidas, que são desproporcionalmente atingidos (Aagaard-Hansen, Nombela \& Alvar, 2010). A migração pode levar à dificuldade de acesso aos serviços de saúde e, no caso da hanseníase, ocorrer um diagnóstico tardio (Monteiro et al., 2017).

Embora tenha sido observada uma tendência de redução temporal de diferentes indicadores de hanseníase no Brasil na 
última década, ressalta-se que a doença ainda apresenta elevada magnitude e persiste como um grave problema de saúde pública no país. Um estudo realizado em 2009 identificou 10 principais clusters de hanseníase, que incluíam 1.173 municípios responsáveis por 53,5\% dos casos novos detectados entre 1980 e 2007 (Penna, Oliveira \& Penna, 2009). Um relatório da Organização Mundial da Saúde evidenciou que para o triênio de 2011-2013 houve uma redução do número de municípios nos clusters (621 municípios). Contudo, foi estimado um risco relativo três a oito vezes maior para estas áreas em relação ao risco médio, que eram concentradas em seis estado brasileiros (WHO, 2013). O estado de Alagoas não aparece listado como área de cluster em nenhum dos estudos citados, no entanto, o presente estudo evidenciou a existência de clusters locais, o que reforça o aparecimento e persistência temporal e geográfica de piores indicadores da hanseníase para o estado.

Teixeira (2020) conduziu uma análise espacial da pobreza no estado de Alagoas, comparando os dados dos censos de 2000 e 2010. Foi observado que municípios com alto índice de pobreza estavam adjacentes a municípios com características similares; da mesma forma, aqueles com baixa pobreza tinham limites geográfico com municípios em situações semelhantes. Observou-se ainda que os municípios mais pobres estavam circundados por municípios com baixos valores de renda per capita, baixa urbanização e com altos níveis de concentração de renda, medida pelo Índice de Gini. Avaliando o Indicador Local de Associação Espacial (LISA) do presente estudo, verifica-se que nesse período (2000 para 2010) o número de municípios no cluster alto-alto aumenta de 12 para 15, concentrando-se nas mesorregiões do Sertão e Agreste alagoano.

A mesorregião do Sertão de Alagoas é formada por 26 municípios e, embora seja uma região de baixa densidade populacional $\left(49,34 \mathrm{hab} / \mathrm{km}^{2}\right)$, apresenta valores de indicadores socioeconômicos abaixo da média geral do estado. A taxa média de pobreza dos municípios da mesorregião do Sertão foi estimada em 63,2\% no Censo de 2010, que foi 37,6\% superior à taxa média de pobreza do estado de Alagoas (45,9\%) (Teixeira, 2020). Além disso, os municípios dessa mesorregião são caracterizados por um clima semiárido, com baixos índices de precipitação pluviométrica anual, ou seja, grandes períodos de seca, visto em sua vegetação de caatinga ou de transição. Estas condições climáticas dificultam ainda mais as condições socioeconômicas das pessoas residentes nestes municípios que, muitas vezes, têm maior atividade econômica voltada para pecuária e agricultura familiar, agravando suas condições de vulnerabilidade social, pobreza e maior exposição à hanseníase.

$\mathrm{O}$ perfil dos casos observados nos municípios alagoanos, principalmente relacionado às formas mais graves da doença, aponta mais uma questão a ser discutida. A maior proporção de casos MB é um indicador de diagnóstico tardio e está relacionado às áreas de maior risco para transmissão (Gaschignard et al., 2016). Adicionalmente, este indicador é fundamental para as ações de planejamento dos municípios e logística de abastecimento de PQT nas unidades dispensadoras (Brasil, 2016). O grau de incapacidade descreve o comprometimento gerado pela hanseníase e é um importante indicador para avaliar o impacto do tratamento na vida do paciente e a qualidade da atenção prestada, assim a proporção de casos novos de hanseníase com grau 2 de incapacidade física no diagnóstico, reflete a dificuldade das ações de detecção precoce dos casos (Brasil, 2016; Raposo et al., 2018). Este indicador sugere a necessidade de novos subsídios para as ações preventivas e de tratamento das incapacidades principalmente no pós-alta, visto que, as sequelas incapacitantes são consideradas perdas, físicas e sociais, irreparáveis em muitos casos (Van Brakel et al., 2012).

Este cenário demonstra as grandes dificuldades enfrentadas para o controle da hanseníase no Brasil. A nova 'Estratégia global para a hanseníase (2021-2030): Towards zero leprosy' apontou os atuais desafios para alcançar um "mundo livre de hanseníase" que vão desde o atraso na deteç̧ão de casos novos, acesso limitado, sistemas de informação inconsistentes, pesquisas escassas, migrações até as questões relacionadas ao estigma entre outras dificuldades a serem enfrentadas. Mesmo diante destas dificuldades a nova estratégia global para a hanseníase propõe atingir zero de infecção e de doença, incapacidade, estigma e discriminação, sobretudo com a interrupção da cadeia de transmissão (WHO, 2021). Esse será um importante avanço no combate à hanseníase, contudo, estas metas e objetivos para serem alcançados devem superar os desafios, sobretudo em países de dimensões continentais e com grandes desigualdades sociais como o Brasil. 
Ressalta-se que há quase 100 anos o desenvolvimento social foi uma estratégia-chave e apontado como prioritário para o alcance do controle da hanseníase em países desenvolvidos (Lie, 1929). Portanto, a melhoria das condições socioeconômicas e redução das iniquidades sociais e de saúde continuam sendo essenciais para reduzir a carga de hanseníase em países de média e baixa renda, como o Brasil. Nessa perspectiva, as reflexões relativas aos elementos de vulnerabilidade social devem ser integradas nas agendas de enfrentamento da hanseníase no país. Para além do setor clínico da saúde, tais agendas deverão considerar espectros maiores dos determinantes sociais envolvidos no atual quadro epidemiológico da hanseníase e as ações de prevenção, diagnóstico e tratamento oportuno devem ser compreendidas como essenciais no cuidado com os indivíduos, famílias e comunidades.

Embora esse estudo tenha tido uma grande oportunidade de apresentar dados de indicadores da hanseníase para uma década no estado de Alagoas, nossas análises apresentam algumas limitações. Por se tratar de um estudo ecológico, há possibilidade de ocorrência de falácia ecológica, e os resultados aqui apresentados não podem ser interpretados a nível individual. Além disso, não foi realizada uma análise de associação entre as taxas de hanseníase e indicadores socioeconômicos locais, para mensurar estimativas pontuais de maior risco de hanseníase em áreas de menor nível socioeconômico. Por fim, não havia disponibilidade de outras variáveis importantes, tais como cobertura dos serviços de saúde e ações/estratégias dos programas de controles locais, para melhor compreender a situação epidemiológica de Alagoas.

\section{Conclusão}

A TDCN geral do estado de Alagoas entre os anos de 2010 e 2019 foi semelhante às médias nacionais estimadas para o período. Muitos municípios apresentaram áreas de alta e superendemia, sendo o município de Santana do Ipanema que apresentou a maior média. A análise espacial da distribuição da hanseníase no estado de Alagoas identificou clusters na faixa oeste-noroeste, região de maior vulnerabilidade socioeconômica do estado.

Esses achados reforçam a importância de análises espaciais como uma ferramenta importante para identificação de áreas de maior magnitude da endemia. Além disso, pode ser um forte indicador de áreas para as ações estratégias de vigilância e controle da hanseníase. Como discutido, essa vigilância deve incluir não apenas aspectos epidemiológicos e operacionais dos serviços de saúde, mas também os fatores de risco relacionados às condições sociais, econômicas e sanitárias dos indivíduos sob exposição diferencial ao $M$. leprae. Recomenda-se o planejamento de ações preventivas, de busca ativa de casos e avaliação de contatos nas áreas identificadas.

\section{Referências}

Aagaard-Hansen, J., Nombela, N., \& Alvar, J. (2010). Population movement: a key factor in the epidemiology of neglected tropical diseases. Tropical Medicine \& International Health, 15(11), 1281-1288.

Almeida, E. (2012). Econometria espacial. Alínea.

Anselin, L. (2005). Exploring spatial data with GeoDaTM: a workbook. Center for spatially integrated social science.

Bailey, T. C., \& Gatrell, A. C. (1995). Interactive spatial data analysis. 413(8).Longman Scientific \& Technical.

Barbosa, C. C., Bonfim, C. V. D., de Brito, C. M. G., Ferreira, A. T., Gregório, V. R. D. N., de Oliveira, A. L. S., \& de Medeiros, Z. M. (2018). Spatial analysis of reported new cases and local risk of leprosy in hyper-endemic situation in Northeastern Brazil. Tropical Medicine \& International Health, 23(7), $748-757$.

Brasil. (2019). Ministério da Saúde. Departamento de Informátoca do SUS. Informações de Saúde. Epidemiológicas e de Morbidade. Hanseníase. http://www2.datasus.gov.br/DATASUS/index.php?area=0203\&id=31032752. Brasília, DF. 2019.

Brasil. (2021). Ministério da Saúde. Sala de Apoio à Gestão Estratégica. Situação de Saúde. Indicadores de Morbidade. Hanseníase. https://sage.saude.gov.br/\# (accessed Feb 12, 2021).

Brasil. (2016). Ministério da Saúde. Secretaria de Vigilância em Saúde. Diretrizes para vigilância, atenção e eliminacao da hanseníase como problema de saúde pública: manual técnico-operacional. 
Ferreira, F. R., \& Nascimento, L. F. C. (2019). Spatial approach of leprosy in the State of São Paulo, 2009-2012. Anais brasileiros de dermatologia, 94(1), 3741.

Freitas, L. R. S. D., Duarte, E. C., \& Garcia, L. P. (2017). Análise da situação epidemiológica da hanseníase em uma área endêmica no Brasil: distribuição espacial dos períodos 2001-2003 e 2010-2012. Revista Brasileira de Epidemiologia, 20, 702-713.

Gaschignard, J., Grant, A. V., Thuc, N. V., Orlova, M., Cobat, A., Huong, N. T., \& Alcais, A. (2016). Pauci-and multibacillary leprosy: two distinct, genetically neglected diseases. PLoS neglected tropical diseases, 10(5), e0004345.

Kerr-Pontes, L. R., Barreto, M. L., Evangelista, C. M., Rodrigues, L. C., Heukelbach, J., \& Feldmeier, H. (2006). Socioeconomic, environmental, and behavioural risk factors for leprosy in North-east Brazil: results of a case-control study. International journal of epidemiology, 35(4), 994-1000.

Leon, K. E., Jacob, J. T., Franco-Paredes, C., Kozarsky, P. E., Wu, H. M., \& Fairley, J. K. (2016, April). Delayed diagnosis, leprosy reactions, and nerve injury among individuals with Hansen's disease seen at a United States clinic. In Open Forum Infectious Diseases (Vol. 3, No. 2, p. ofw063). Oxford University Press.

Lie, H. P. (1929). Why is leprosy decreasing in Norway?. Transactions of The Royal Society of Tropical Medicine and Hygiene, 22(4).

Lockwood, D. N., \& Saunderson, P. R. (2012). Nerve damage in leprosy: a continuing challenge to scientists, clinicians and service providers. International health, 4(2), 77-85.

Monteiro, L. D., Martins-Melo, F. R., Brito, A. L., Alencar, C. H., \& Heukelbach, J. (2015). Spatial patterns of leprosy in a hyperendemic state in Northern Brazil, 2001-2012. Revista de Saúde Pública, 49, 84.

Monteiro, L. D., Mota, R. M. S., Martins-Melo, F. R., Alencar, C. H., \& Heukelbach, J. (2017). Determinantes sociais da hanseníase em um estado hiperendêmico da região Norte do Brasil. Revista de Saúde Pública, 51, 70.

Murto, C., Chammartin, F., Schwarz, K., da Costa, L. M. M., Kaplan, C., \& Heukelbach, J. (2013). Patterns of migration and risks associated with leprosy among migrants in Maranhao, Brazil. PLoS neglected tropical diseases, 7(9), e2422.

Lencucha, R., Drope, J., Bialous, S. A., \& Richter, A. P. (2017). Institutions and the implementation of tobacco control in Brazil. Cadernos de Saúde Pública, 33, e00168315.

Nery, J. S., Ramond, A., Pescarini, J. M., Alves, A., Strina, A., Ichihara, M. Y., Penna, M. L. F., Smeeth L., Rodrigues, L. C., Barreto, M. L., Brickley, E. B., Penna, G. O. (2019). Socioeconomic determinants of leprosy new case detection in the 100 Million Brazilian Cohort: a population-based linkage study. The Lancet Global Health, 7(9), e1226-e1236.

Penna, M. L. F., Reydeoliveira, M. V., \& Penna, G. O. (2009). The epidemiological behaviour of leprosy in Brazil. Leprosy review, 80(3), 332-345.

Pescarini, J. M., Strina, A., Nery, J. S., Skalinski, L. M., Andrade, K. V. F., Penna, M. L. F., Brickley, E. B., Rodrigues, L. C., Barreto, M. L., Penna, G. O. (2018). Socioeconomic risk markers of leprosy in high-burden countries: A systematic review and meta-analysis. PLoS neglected tropical diseases, 12(7), e0006622.

Pescarini, J. M., Williamson, E., Nery, J. S., Ramond, A., Ichihara, M. Y. Fiaccone, R. L., Yuri, M., Penna, M. L. F., Smeeth, L., Rodrigues, L. C., Penna, G. O., Brickley, E. B., Barreto, M. L. (2020). Effect of a conditional cash transfer programme on leprosy treatment adherence and cure in patients from the nationwide 100 Million Brazilian Cohort: a quasi-experimental study. The Lancet Infectious Diseases, 20(5), 618-627.

Rao, P. N. (2017). Global leprosy strategy 2016-2020: Issues and concerns. Indian J Dermatol Venereol Leprol; 83, 4-6.

Raposo, M. T., Reis, M. C., de Queiroz Caminha, A. V., Heukelbach, J., Parker, L. A., Pastor-Valero, M., \& Nemes, M. I. B. (2018). Grade 2 disabilities in leprosy patients from Brazil: Need for follow-up after completion of multidrug therapy. PLoS neglected tropical diseases, 12(7), e0006645.

Schneider, P. B., \& Freitas, B. H. B. M. D. (2018). Leprosy trends in children under 15 years of age in Brazil, 2001-2016. Cadernos de Saúde Pública, 34(3).

Talhari, C., Talhari, S., Penna, G. O. (2015). Clinical aspects of leprosy. Clin Dermatol, 33: 26-37.

Teixeira, K. H. (2020). Uma análise espacial da pobreza no Estado de Alagoas. Redes; 25: 2668-2692.

WHO. (2010). First WHO report on neglected tropical diseases: working to overcome the global impact of neglected tropical diseases. World Heal Organ 2010. $10.1177 / 1757913912449575$.

WHO. (2020). Global leprosy (Hansen disease) update, 2019: time to step-up prevention initiatives. Wkly Epidemiol Rec; 95: 417-40.

WHO. (2021). Global Leprosy (Hansen’s Disease) Strategy 2021-2030: Towards Zero Leprosy.

WHO. (2013). Leprosy elimination. Cluster analysis of the overall detection rate of leprosy in Brazil for the triennium 2011-2013. Disponível em: https://www.who.int/lep/resources/Cluster_analysis/en/.

Wilder-Smith, E. P., \& Van Brakel, W. H. (2008). Nerve damage in leprosy and its management. Nat Clin Pract Neurol, 4: 656-63.

Withington, S. G., Joha, S., Baird, D., Brink, M., Brink, J. (2003). Assessing socio-economic factors in relation to stigmatization, impairment status, and selection for socio-economic rehabilitation: a 1-year cohort of new leprosy cases in north Bangladesh. Leprosy Review, 74: 120-32.

Van Brakel, W. H., Sihombing, B., Djarir, H., Beise, K., Kusumawardhani, L., Yulihane, R. \& Wilder-Smith, A. (2012). Disability in people affected by leprosy: the role of impairment, activity, social participation, stigma and discrimination. Global health action, 5(1), 18394. 
Research, Society and Development, v. 10, n. 5, e48510514962, 2021

(CC BY 4.0) | ISSN 2525-3409 | DOI: http://dx.doi.org/10.33448/rsd-v10i5.14962

Visschedijk, J., Borek, J. V. de, Eggens, H., Lever, P., Beers, S. V., Klatser, P. (2000). Review: Mycobacterium leprae - millennium resistant! Leprosy control on the threshold of a new era. Tropical Medicine International Health, 5: 388-99. 